\title{
Uncertainty principle and geometry of the infinite Grassmann manifold
}

\author{
Esteban Andruchow and Gustavo Corach
}

August 3, 2018

\begin{abstract}
We study the pairs of projections

$$
P_{I} f=\chi_{I} f, \quad Q_{J} f=\left(\chi_{J} \hat{f}\right)\left\llcorner, f \in L^{2}\left(\mathbb{R}^{n}\right),\right.
$$

where $I, J \subset \mathbb{R}^{n}$ are sets of finite Lebesgue measure, $\chi_{I}, \chi_{J}$ denote the corresponding characteristic functions and ", denote the Fourier-Plancherel transformation $L^{2}\left(\mathbb{R}^{n}\right) \rightarrow L^{2}\left(\mathbb{R}^{n}\right)$ and its inverse. These pairs of projections have been widely studied by several authors in connection with the mathematical formulation of Heisenberg's uncertainty principle. Our study is done from a differential geometric point of view. We apply known results on the Finsler geometry of the Grassmann manifold $\mathcal{P}(\mathcal{H})$ of a Hilbert space $\mathcal{H}$ to establish that there exists a unique minimal geodesic of $\mathcal{P}(\mathcal{H})$, which is a curve of the form
\end{abstract}

$$
\delta(t)=e^{i t X_{I, J}} P_{I} e^{-i t X_{I, J}}
$$

which joins $P_{I}$ and $Q_{J}$ and has length $\pi / 2$. As a consequence we obtain that if $H$ is the logarithm of the Fourier-Plancherel map, then

$$
\left\|\left[H, P_{I}\right]\right\| \geq \pi / 2 \text {. }
$$

The spectrum of $X_{I, J}$ is denumerable and symmetric with respect to the origin, it has a smallest positive eigenvalue $\gamma\left(X_{I, J}\right)$ which satisfies

$$
\cos \left(\gamma\left(X_{I, J}\right)\right)=\left\|P_{I} Q_{J}\right\| .
$$

2010 MSC: 58B20, 47B15, 42A38, 47A63.

Keywords: Projections, pairs of projections, Grasmann manifold, uncertainty principle.

\section{Introduction}

Consider the following example:

Example 1.1. Let $I, J \subset \mathbb{R}^{n}$ be Lebesgue-measurable sets of finite measure. Let $P_{I}, Q_{J}$ be the projections in $L^{2}\left(\mathbb{R}^{n}, d x\right)$ given by

$$
P_{I} f=\chi_{I} f \quad \text { and } \quad Q_{J} f=\left(\chi_{J} \hat{f}\right)
$$


where $\chi_{L}$ denotes the characteristic function of the set $L$. Equivalently, denoting by $U_{\mathcal{F}}$ the Fourier transformation regarded as a unitary operator acting in $L^{2}\left(\mathbb{R}^{n}, d x\right)$ and by $M_{\varphi}$ the multiplication by $\varphi$, then

$$
P_{I}=M_{\chi_{I}} \text { and } Q_{J}=U_{\mathcal{F}}^{*} P_{J} U_{\mathcal{F}} \text {. }
$$

The operator $P_{I} Q_{J}$ is Hilbert-Schmidt (see for instance [11], Lemma 2).

An intuitive formulation of Heisenberg's uncertainty principle says that a nonzero function and its Fourier transform cannot be (simultaneously) sharply localized (see [13], page 207). We give more precision to this statement below ( see for instance [11], page 906).

According to Folland and Sitaram [13], the idea of using projections $P_{I}$ and $Q_{J}$ to obtain a form of the uncertainty principle is due to Fuchs [14], and it was developed later in a series of papers by Landau, Pollack and Slepian [20], [21], [25]. See the survey by Folland and Sitaram $[13]$.

Donoho and Stark [11] proved that if $I, J \subset \mathbb{R}^{n}$ with finite Lebesgue measure and $f \in L^{2}\left(\mathbb{R}^{n}\right)$ with $\|f\|_{2}=1$ satisfy that

$$
\int_{\mathbb{R}^{n}-I}|f(t)|^{2} d t<\epsilon_{I} \text { and } \int_{\mathbb{R}^{n}-J}|\hat{f}(w)|^{2} d w<\epsilon_{J}
$$

then

$$
|I||J| \geq\left(1-\left(\epsilon_{I}+\epsilon_{J}\right)\right)^{2} .
$$

Donoho and Stark showed several applications of these ideas to signal processing (and the obstruction to the existence of an instantaneous frequency). Smith [26] generalized these results to a locally compact abelian group $G$ where $I \subset G$ and $J \subset \hat{G}$, the dual group of $G$. The books by Havin and Jöricke [17], Hogan and Lakey [18], and Gröchenig [15] among many others, contain further applications, generalizations and history of the different uncertainty principles.

By an elementary computation using Fubini's theorem, Donoho and Stark prove that

$$
\left\|P_{I} Q_{J}\right\|_{H S}=\sqrt{|I||J|},
$$

where \|\|$_{H S}$ is Hilbert-Schmidt norm. Next they prove that

$$
\left\|P_{I} Q_{J}\right\| \geq 1-\epsilon_{I}-\epsilon_{J}
$$

The fact that $\left\|P_{I} Q_{J}\right\| \leq\left\|P_{I} Q_{J}\right\|_{H S}$ is well known.

They argue that any bound $c$ such that

$$
\left\|P_{I} Q_{J}\right\| \leq c<1
$$

is an expression of the uncertainty principle ([11], page 912).

Denote by $\mathcal{P}(\mathcal{H})$ the set of orthogonal projections of the Hilbert space $\mathcal{H}$, also called the Grassmann manifold of $\mathcal{H}$. It is indeed a differentiable manifold of $\mathcal{B}(\mathcal{H})$ (also in the infinite dimensional setting), with rich geometric structure (see for instance [24] or [7]). The pairs $\left(P_{I}, Q_{J}\right)$ might be put in the broader context of the sets

$$
\mathcal{C}=\{(P, Q): P, Q \text { are orthogonal projections and } P Q \text { is compact }\} .
$$

This set is a $C^{\infty}$-submanifold of $\mathcal{P}(\mathcal{H}) \times \mathcal{P}(\mathcal{H})$. 
An application of these geometrical results facts is a form of the uncertainty principle (see Theorem 3.6 below).

Let us describe the content of the paper.

In Section 2 we recall the known facts on the geometry of $\mathcal{P}(\mathcal{H})$. In section 3 we apply known results [24], [7], [2] on the Finsler geometry of the Grassmann manifold of $\mathcal{H}$ to the special case of pairs $P_{I}, Q_{J}$. We prove that there exists a unique minimal geodesic of the Grassmann manifold of length $\pi / 2$ which joins $P_{I}$ and $Q_{J}$. That is, there exists a unique selfadjoint operator $X_{I, J}$ of norm $\pi / 2$, which is co-diagonal with respect both to $P_{I}$ and $Q_{J}$, such that

$$
e^{i X_{I, J}} P_{I} e^{-i X_{I, J}}=Q_{J} .
$$

The spectrum of the operator $X_{I, J}$ is denumerable and symmetric with respect to the origin. The smallest positive eigenvalue $\gamma\left(X_{I, J}\right)$ verifies

$$
\cos \left(\gamma\left(X_{I, J}\right)\right)=\left\|P_{I} Q_{J}\right\|
$$

As a consequence from the fact that the minimal geodesic has length $\pi / 2$, we prove that if $H$ is the logarithm of the Fourier transform in $L^{2}\left(\mathbb{R}^{n}\right)$, and $I \subset \mathbb{R}^{n}$ is a set of finite Lebesgue measure, then

$$
\left\|\left[H, P_{I}\right]\right\|=\left\|\left[H, Q_{I}\right]\right\| \geq \pi / 2 .
$$

In Section 4 we show that for any pair of sets $I, J \subset \mathbb{R}^{n}$ of finite measure, one has

$$
N\left(P_{I}\right)+N\left(Q_{J}\right)=L^{2}\left(\mathbb{R}^{n}\right),
$$

where the sum is non-direct (the subspaces have infinite dimensional intersection).

\section{Basic properties}

\subsection{Halmos decomposition}

Let $\mathcal{H}$ be a Hilbert space, $\mathcal{B}(\mathcal{H})$ the algebra of bounded linear operators in $\mathcal{H}, \mathcal{K}(\mathcal{H})$ the ideal of compact operators and $\mathcal{P}(\mathcal{H})$ the set of selfadjoint (orthogonal) projections, and $\mathcal{P}_{\infty}(\mathcal{H})$ the subset of projections whose nullspaces and ranges have infinite dimension.

A tool that will be useful in the study of the pairs $P_{I}, Q_{J}$ is Halmos decomposition [16], which is the following orthogonal decomposition of $\mathcal{H}$ : given a pair of projections $P$ and $Q$, consider

$$
\mathcal{H}_{11}=R(P) \cap R(Q), \quad \mathcal{H}_{00}=N(P) \cap N(Q), \quad \mathcal{H}_{10}=R(P) \cap N(Q), \quad \mathcal{H}_{01}=N(P) \cap R(Q)
$$

and $\mathcal{H}_{0}$ the orthogonal complement of the sum of the above. This last subspace is usually called the generic part of the pair $P, Q$. Note also that

$$
N(P-Q)=\mathcal{H}_{11} \oplus \mathcal{H}_{00}, \quad N(P-Q-1)=\mathcal{H}_{10} \text { and } N(P-Q+1)=\mathcal{H}_{01},
$$

so that the generic part depends in fact of the difference $P-Q$.

Halmos proved that there is an isometric isomorphism between $\mathcal{H}_{0}$ and a product Hilbert space $\mathcal{L} \times \mathcal{L}$ such that in the above decomposition (putting $\mathcal{L} \times \mathcal{L}$ in place of $\mathcal{H}_{0}$ ), the projections are

$$
P=1 \oplus 0 \oplus 1 \oplus 0 \oplus\left(\begin{array}{ll}
1 & 0 \\
0 & 0
\end{array}\right)
$$


and

$$
Q=1 \oplus 0 \oplus 0 \oplus 1 \oplus\left(\begin{array}{cc}
C^{2} & C S \\
C S & S^{2}
\end{array}\right)
$$

where $C=\cos (X)$ and $S=\sin (X)$ for some operator $0<X \leq \pi / 2$ in $\mathcal{L}$ with trivial nullspace.

Aparently, the pair $(P, Q)$ belongs to $\mathcal{C}$ if and only if $\mathcal{H}_{11}$ is finite dimensional and $C=\cos (X)$ is compact.

Remark 2.1. If $(P, Q) \in \mathcal{C}$, then the spectral resolution of $X$ can be easily described. Since $0<\cos (X)$ is compact, it follows that

$$
X=\sum_{n} \gamma_{n} P_{n}+\frac{\pi}{2} E
$$

where $0<\gamma_{n}<\pi / 2$ is an increasing (finite or infinite) sequence. For all $n$, $\operatorname{dim} R\left(P_{n}\right)<\infty$, and

$$
R(E) \oplus\left(\oplus_{n \geq 1} R\left(P_{n}\right)\right)=\mathcal{L} .
$$

\subsection{Finsler geometry of the Grassmann manifold of $\mathcal{H}$}

Let us recall some basic facts on the differential geometry of the set $\mathcal{P}(\mathcal{H})$ (see for instance [7], $[24],[2])$.

1. The space $\mathcal{P}(\mathcal{H})$ is a homogeneous space under the action of the unitary group $\mathcal{U}(\mathcal{H})$ by inner conjugation: if $U \in \mathcal{U}(\mathcal{H})$ and $P \in \mathcal{P}(\mathcal{H})$, the action is given by

$$
U \cdot P=U P U^{*}
$$

This action is locally transitive: it is well known that two projections $P_{1}, P_{2}$ such that $\left\|P_{1}-P_{2}\right\|<1$, are conjugate. Therefore, since the unitary group $\mathcal{U}(\mathcal{H})$ is connected, the orbits of the action coincide with the connected components of $\mathcal{P}(\mathcal{H})$, which are: for $n \in \mathbb{N}, \mathcal{P}_{n, \infty}(\mathcal{H})$ (projections of nullity $\left.n\right), \mathcal{P}_{\infty, n}(\mathcal{H})$ (projections of rank $n$ ) and $\mathcal{P}_{\infty}(\mathcal{H})$ (projections of infinite rank and nullity). These components are $C^{\infty}$-submanifolds of $\mathcal{B}(\mathcal{H})$.

2. There is a natural linear connection in $\mathcal{P}(\mathcal{H})$. If $\operatorname{dim} \mathcal{H}<\infty$, it is the Levi-Civita connection of the Riemannian metric which consists of considering the Frobenius inner product at every tangent space. It is based on the diagonal / co-diagonal decomposition of $\mathcal{B}(\mathcal{H})$. To be more specific, given $P_{0} \in \mathcal{P}(\mathcal{H})$, the tangent space of $\mathcal{P}(\mathcal{H})$ at $P_{0}$ consists of all selfadjoint co-diagonal matrices (in terms of $P_{0}$ ). The linear connection in $\mathcal{P}(\mathcal{H})$ is induced by a reductive structure, where the horizontal elements at $P_{0}$ (in the Lie algebra of $\mathcal{U}(\mathcal{H})$ : the space of antihermitian elements of $\mathcal{B}(\mathcal{H})$ ) are the co-diagonal antihermitian operators. The geodesics of $\mathcal{P}$ which start at $P_{0}$ are curves of the form

$$
\delta(t)=e^{i t X} P_{0} e^{-i t X}
$$

with $X^{*}=X$ co-diagonal with respect to $P_{0}$. Observe that $X$ is co-diagonal with respect to every $P_{t}=\delta(t)$. It was proved in [24] that if $P_{0}, P_{1} \in \mathcal{P}(\mathcal{H})$ satisfy $\left\|P_{0}-P_{1}\right\|<1$, then there exists a unique geodesic (up to reparametrization) joining $P_{0}$ and $P_{1}$. This condition is not necessary for the existence of a unique geodesic. 
3. There exists a unique geodesic joining two projections $P$ and $Q$ if and only if

$$
R(P) \cap N(Q)=N(P) \cap R(Q)=\{0\},
$$

$($ see $[2])$.

4. If $\mathcal{H}$ is infinite dimensional, the Frobenius metric is not available. However, if one endows each tangent space of $\mathcal{P}(\mathcal{H})$ with the usual norm of $\mathcal{B}(\mathcal{H})$, one obtains a continuous (non regular) Finsler metric,

$d\left(P_{0}, P_{1}\right)=\inf \left\{\ell(\gamma): \gamma\right.$ a continuous piecewise smooth curve in $\mathcal{P}(\mathcal{H})$ joining $P_{0}$ and $\left.P_{1}\right\}$ where $\ell(\gamma)$ denotes the length of $\gamma$ (parametized in the interval $I$ ):

$$
\ell(\gamma)=\int_{I}\|\dot{\gamma}(t)\| d t
$$

In [24] it was shown that the geodesics (1) remain minimal among their endpoints for all $t$ such that

$$
|t| \leq \frac{\pi}{2\|X\|}
$$

It can be shown that $d\left(P_{0}, P_{1}\right)<\pi / 2$ if and only if $\left\|P_{0}-P_{1}\right\|<1$. In other words, $\left\|P_{0}-P_{1}\right\|=1$ if and only if $d\left(P_{0}, P_{1}\right)=\pi / 2$.

\section{Geometry of the pairs $P_{I}, Q_{J}$}

Lenard proved in $[22]$ that the projections $P_{I}, Q_{J} \in \mathcal{P}\left(L^{2}\left(\mathbb{R}^{n}, d x\right)\right)$ defined in Example (1.1), satisfy

$$
R\left(P_{I}\right) \cap N\left(Q_{J}\right)=R\left(Q_{J}\right) \cap N\left(P_{I}\right)=\{0\} .
$$

Moreover, $\left\|P_{I}-Q_{J}\right\|=1$.

Therefore one obtains the following:

Theorem 3.1. Let $I, J$ be measurable subsets of $\mathbb{R}^{n}$ of finite measure, and $P_{I}, Q_{J}$ the above projections. Then there exists a unique selfadjoint operator $X_{I, J}$ satisfying:

1. $\left\|X_{I, J}\right\|=\pi / 2$.

2. $X_{I, J}$ is $P_{I}$ and $Q_{J}$ co-diagonal. In other words, $X_{I, J}$ maps functions in $L^{2}\left(\mathbb{R}^{n}, d x\right)$ with support in $I$ to functions with support in $\mathbb{R}^{n}-I$, and functions such that $\hat{f}$ has support in $J$ to functions such that the Fourier transform has support in $\mathbb{R}^{n}-J$.

3. $e^{i X_{I, J}} P_{I} e^{-i X_{I, J}}=Q_{J}$.

4. If $P(t), t \in[0,1]$ is a smooth curve in $\mathcal{P}(\mathcal{H})$ with $P(0)=P_{I}$ and $P(1)=Q_{J}$, then

$$
\ell(P)=\int_{0}^{1}\|\dot{P}(t)\| d t \geq \pi / 2 .
$$


Proof. By the condition (2) above $([22])$, it follows from [2] that there exists a unique minimal geodesic of $\mathcal{P}(\mathcal{H})$, of the form

$$
\delta_{I, J}(t)=e^{i t X_{I, J}} P_{I} e^{i t X_{I, J}}
$$

with $X_{I, J}^{*}=X_{I, J}$ co-doagonal with respect to $P_{I}$ (and $Q_{J}$ ) such that

$$
\delta_{I, J}(1)=Q_{J} .
$$

Condition 4. above is the minimality property of $\delta_{I, J}$. Finally, the fact that $\left\|P_{I}-Q_{J}\right\|=1$ means that $\left\|X_{I, J}\right\|=\pi / 2$.

Remark 3.2. It is known [13] that $\lambda_{1}=\left\|P_{I} Q_{J} P_{I}\right\|=\left\|P_{I} Q_{J}\right\|^{2}<1$, and moreover $\sqrt{\lambda_{1}}$ equals the cosine of the angle between the subspaces $R\left(P_{I}\right)$ and $R\left(Q_{J}\right)$.

One can also relate this number $\lambda_{1}$ with the operator $X_{I, J}$. Using Halmos decomposition (recall that it consists only of $\mathcal{H}_{00}$ and the generic part $\mathcal{H}_{0}$ in this case),

$$
P_{I} Q_{J} P_{I}=0 \oplus\left(\begin{array}{cc}
C^{2} & 0 \\
0 & 0
\end{array}\right)
$$

and thus $\lambda_{1}=\|\cos (X)\|^{2}$. We shall see below that the spectrum of $X$ is a strictly increasing sequence of positive eigenvalues $\gamma_{n} \rightarrow \pi / 2$, with finite multiplicity. Moreover, since $P_{I} Q_{J} P_{I}$ belongs to $\mathcal{B}_{1}(\mathcal{H})$, it follows that $C \in \mathcal{B}_{2}(\mathcal{L})$. Thus

$$
\left\{\cos \left(\gamma_{n}\right)\right\} \in \ell^{2} .
$$

For a given $P \in \mathcal{P}(\mathcal{H})$, let $\mathcal{A}_{P}$ be

$$
\mathcal{A}_{P}=\{X \in \mathcal{B}(\mathcal{H}):[X, P] \text { is compact }\} .
$$

Apparently $\mathcal{A}_{P}$ is a $\mathrm{C}^{*}$-algebra.

Theorem 3.3. Let $I, J$ be measurable subsets of $\mathbb{R}^{n}$ of finite Lebesgue measure.

1. The selfadjoint operator $X_{I, J}$ has closed infinite dimensional range, in particular it is not compact.

2. Let $I_{0}$ be another measurable set with finite measure such that $\left|I \cap I_{0}\right|=0$, and let $P_{0}=P_{I_{0}}$. Then, the commutant $\left[X_{I, J}, P_{0}\right]$ is compact.

Proof. Easy matrix computations ([2]) show that, in the decomposition $\mathcal{H}=\mathcal{H}_{00} \oplus(\mathcal{L} \times \mathcal{L})$, $X_{I, J}$ is of the form

$$
X_{I, J}=0 \oplus\left(\begin{array}{cc}
0 & -i X \\
i X & 0
\end{array}\right) .
$$

Note that the spectrum of this operator is symmetric with respect to the origin. Indeed, if $V$ equals the symmetry

$$
V=1 \oplus\left(\begin{array}{ll}
0 & 1 \\
1 & 0
\end{array}\right),
$$

then apparently $V X_{I, J} V=-X_{I, J}$. Also note that

$$
X_{I, J}^{2}=0 \oplus\left(\begin{array}{cc}
X^{2} & 0 \\
0 & X^{2}
\end{array}\right) .
$$


Therefore the spectrum of $X_{I, J}$ is

$$
\sigma\left(X_{I, J}\right)=\{0\} \cup\left\{\gamma_{n}: n \geq 1\right\} \cup\left\{-\gamma_{n}: n \geq 1\right\},
$$

with 0 of infinite multiplicity, and the multiplicity of $\gamma_{n}$ equal to the multiplicity of $-\gamma_{n}$, and finite. What matters here, is that the set $\left\{\gamma_{n}: n \geq 1\right\}$ is infinite, and is therefore an increasing sequence converging to $\pi / 2$. This holds because otherwise, the operator $C$ would have finite rank, and therefore $P_{I} Q_{J} P_{I}$ would be of finite rank, which is not the case (see [22]). Thus $X_{I, J}$ has closed range. of infinite dimension.

Note that $P_{I}$ and $Q_{J}$ satisfy that $P_{I} P_{0}=0$ and $Q_{J} P_{0}=Q_{J} P_{I_{0}}$ is compact, and therefore $P_{I}, Q_{J} \in \mathcal{A}_{P_{0}}$. Thus the symmetries $S_{P_{I}}, S_{Q_{J}}$ belong to $a_{P_{0}}$. Since $S_{Q_{J}}=e^{i 2 X_{I, J}} S_{P_{I}}$, this implies that

$$
e^{i 2 X_{I, J}} \in \mathcal{A}_{P_{0}} .
$$

By the spectral picture of $X_{I, J}$ it is clear that $X_{I, J}$ can be obtained as an holomorphic function of $e^{i 2 X_{I, J}}$. Since $\mathcal{A}_{P_{0}}$ is a $\mathrm{C}^{*}$-algebra, this implies that $X_{I, J} \in \mathcal{A}_{P_{0}}$.

Let us relate the operator $X_{I, J}$ with the mathematical version of the uncertainty principle, according to [11] and [13].

Let $A \in \mathcal{B}(\mathcal{H})$ be an operator with closed range, the reduced minimum modulus $\gamma_{A}$ of $A$ is the positive number

$$
\gamma_{A}=\min \left\{\|A \xi\|: \xi \in N(A)^{\perp},\|\xi\|=1\right\}=\min \{|\lambda|: \lambda \in \sigma(A), \lambda \neq 0\} .
$$

Donoho and Stark [11] underline the role of the number $\left\|Q_{J} P_{I}\right\|$ and consider any constant $c$ such that $\left\|Q_{J} P_{I}\right\| \leq c$ a manifestation of the (mathematical) uncertainty principle. By the above Remark, we have:

Corollary 3.4. With the current notations,

$$
\left\|Q_{J} P_{I}\right\|=\cos \left(\gamma_{X_{I . J}}\right) .
$$

Proof. Indeed, in the above description of the spectrum of $X_{I, J}$, the reduced minimum modulus $\gamma_{X_{I . J}}$ of $X_{I, J}$ coincides with $\gamma_{1}$.

Let $X_{I, J}^{0}$ be the restriction of $X_{I, J}$ to the generic part of $P_{I}$ and $Q_{J}$, i.e., its restriction to $N\left(X_{I, J}\right)^{\perp}$. In Halmos decomposition

$$
X_{I, J}^{0}=\left(\begin{array}{cc}
0 & -i X \\
i X & 0
\end{array}\right)
$$

Recall the formula by Donoho and Stark [11]

$$
\left\|P_{I} Q_{J}\right\|_{H S}=|I|^{1 / 2}|J|^{1 / 2}
$$

From the preceeding facts, it also follows:

Corollary 3.5. With the current notations

$$
|I|^{1 / 2}|J|^{1 / 2}=\|\cos (X)\|_{H S}=\frac{1}{\sqrt{2}}\left\|\cos \left(X_{I, J}^{0}\right)\right\|_{H S}=\left\{\sum_{n=1}^{\infty} \frac{1}{2} \cos \left(\gamma_{n}\right)^{2}\right\}^{1 / 2} .
$$


Proof.

$$
|I||J|=\left\|P_{I} Q_{J}\right\|_{H S}^{2}=\operatorname{Tr}\left(P_{I} Q_{J} P_{I}\right)=\operatorname{Tr}\left(C^{2}\right)=\frac{1}{2} \operatorname{Tr}\left(\begin{array}{cc}
C^{2} & 0 \\
0 & C^{2}
\end{array}\right)=\frac{1}{2} \operatorname{Tr}\left(\cos \left(X_{I, J}^{0}\right)^{2}\right) .
$$

This co-diagonal exponent $X_{I, J}$ (with respect both to $P_{I}$ and $Q_{J}$ ) has interesting features when $I=J$ and $|I|<\infty$. In this case denote by $X_{I}=X_{I, I}$; then, we have two unitary operators intertwining $P_{I}$ and $Q_{I}$. Namely, the Fourier transform $U_{\mathcal{F}}$ and the exponential $e^{i X_{I}}$,

$$
U_{\mathcal{F}}^{*} P_{I} U_{\mathcal{F}}=Q_{I}=e^{i X_{I}} P_{I} e^{-i X_{I}} .
$$

Let $H=H^{*}$ be the natural logarithm of the Fourier transform, $e^{i H}=U_{\mathcal{F}}$. Namely, writing $E_{1}$, $E_{-1}, E_{i}$ and $E_{-i}$ the eigenprojections of $U_{\mathcal{F}}$,

$$
H=-\pi E_{-1}+\frac{\pi}{2} E_{i}-\frac{\pi}{2} E_{-i} .
$$

Note that $\|H\|=\pi$. Thus, one obtains a smooth path joining $P_{I}$ and $Q_{I}$ :

$$
\varphi(t)=e^{-i t H} P_{I} e^{i t H} \text {. }
$$

and, apparently, $\varphi(1)=Q_{I}$.

Since the Fourier transform intertwines $P_{I}$ and $Q_{J}$, the norm of its commutant with either of these projections can be regarded as a measure of non commutativity between $P_{I}$ and $Q_{J}$ :

Theorem 3.6. For any Lebesgue measurable set $I \subset \mathbb{R}^{n}$ with $|I|<\infty$, one has

$$
\left\|\left[H, P_{I}\right]\right\|=\left\|\left[H, Q_{I}\right]\right\| \geq \pi / 2 .
$$

Proof. The geodesic $\delta_{I}$ with exponent $X_{I}$ is the shortest curve in $\mathcal{P}(\mathcal{H})$ joining $P_{I}$ and $Q_{I}$. Its length is $\pi / 2$. Then

$$
\pi / 2 \leq \ell(\varphi)=\int_{0}^{1}\|\dot{\varphi}(t)\| d t=\int_{0}^{1}\left\|e^{i t H}\left[H, P_{I}\right] e^{-i t H}\right\| d t=\left\|\left[H, P_{I}\right]\right\| .
$$

Note that

$$
U_{\mathcal{F}}^{*}\left[H, P_{I}\right] U_{\mathcal{F}}=\left[H, U_{\mathcal{F}}^{*} P_{I} U_{\mathcal{F}}\right]=\left[H, Q_{I}\right]
$$

because $U_{\mathcal{F}}$ and $H$ commute.

\section{Remark 3.7.}

1. We may write $H$ in terms of $U_{\mathcal{F}}$ using the well known formulas

$E_{-1}=\frac{1}{4}\left(1-U_{\mathcal{F}}+U_{\mathcal{F}}^{2}-U_{\mathcal{F}}^{3}\right), E_{i}=\frac{1}{4}\left(1-i U_{\mathcal{F}}-U_{\mathcal{F}}^{2}+i U_{\mathcal{F}}^{3}\right), E_{-i}=\frac{1}{4}\left(1+i U_{\mathcal{F}}-U_{\mathcal{F}}^{2}-i U_{\mathcal{F}}^{3}\right)$,

and thus

$$
H=\frac{\pi}{4}\left\{-1+(1+i) U_{\mathcal{F}}-U_{\mathcal{F}}^{2}+(1+i) U_{\mathcal{F}}^{3}\right\}
$$

Then

$$
\left[H, P_{I}\right]=\frac{\pi}{4}\left\{(1+i)\left[U_{\mathcal{F}}, P_{I}\right]-\left[U_{\mathcal{F}}^{2}, P_{I}\right]+(1+i)\left[U_{\mathcal{F}}^{3}, P_{I}\right]\right\}
$$

The inequality in Corollary 3.6 can be written

$$
\left\|(1+i)\left[U_{\mathcal{F}}, P_{I}\right]-\left[U_{\mathcal{F}}^{2}, P_{I}\right]+(1+i)\left[U_{\mathcal{F}}^{3}, P_{I}\right]\right\| \geq 2 .
$$


2. In the special case when the set $I$ is (essentially) symmetric with respect to the origin, $P_{I}$ commutes with $U_{\mathcal{F}}^{2}$, so that

$$
\left[U_{\mathcal{F}}^{2}, P_{I}\right]=0 \text { and }\left[U_{\mathcal{F}}^{3}, P_{I}\right]=\left[U_{\mathcal{F}}, P_{I}\right] U_{\mathcal{F}}^{2}=U_{\mathcal{F}}^{2}\left[U_{\mathcal{F}}, P_{I}\right]
$$

one has

$$
\left[H, P_{I}\right]=\frac{(1+i) \pi}{4}\left[U_{\mathcal{F}}, P_{I}\right]\left(1+U_{\mathcal{F}}^{2}\right)
$$

The operator $U_{\mathcal{F}}^{2} f(x)=f(-x)$ is a symmetry, then $\frac{1}{2}\left(1+U_{\mathcal{F}}^{2}\right)$ is the orthogonal projection $E_{e}$ onto the the subspace of essentially even functions $(f(x)=f(-x)$ a.e.). Then one can write

$$
\left[H, P_{I}\right]=\frac{(1+i) \pi}{2}\left[U_{\mathcal{F}}, P_{I}\right] E_{e}=\frac{(1+i) \pi}{2} E_{e}\left[U_{\mathcal{F}}, P_{I}\right]
$$

Corollary 3.8. Suppose that I is essentially symmetric, with finite measure.

1.

$$
\left\|E_{e}\left[U_{\mathcal{F}}, P_{I}\right]\right\|=\left\|E_{e}\left[U_{\mathcal{F}}, P_{I}\right] E_{e}\right\| \geq \frac{1}{\sqrt{2}}
$$

2.

$$
\left\|E_{e} P_{I}-E_{e} Q_{I}\right\| \geq \frac{1}{\sqrt{2}}
$$

where $E_{e} P_{I}=P_{I} E_{e}$ and $E_{e} Q_{I}=Q_{I} E_{e}$ are orthogonal projections.

Proof. Recall that $E_{e}$ and $U_{\mathcal{F}}$ commute. Then

$$
\begin{gathered}
E_{e}\left[U_{\mathcal{F}}, P_{I}\right] E_{e}=E_{e}\left(U_{\mathcal{F}} P_{I}-P_{I} U_{\mathcal{F}}\right) E_{e}=U_{\mathcal{F}} E_{e}\left(P_{I}-U_{\mathcal{F}}^{*} P_{I} U_{\mathcal{F}}\right) E_{e} \\
=U_{\mathcal{F}} E_{e}\left(P_{I}-Q_{I}\right) E_{e} .
\end{gathered}
$$

where $E_{e}$, as well as $U_{\mathcal{F}}$, and thus also $Q_{I}=U_{\mathcal{F}}^{*} P_{I} U_{\mathcal{F}}$ commute with $E_{e}$.

The ranges of these two orthogonal projections $E_{e} P_{I}$ and $E_{e} Q_{I}$ consist of the elements of $L^{2}$ which are essentially even and vanish (essentially) outside $I$, and the analogous subspace for the Fourier transform.

\section{Spatial properties of $P_{I}$ and $Q_{J}$}

Let us return to the general setting ( $I$ not necessarily equal to $J$ ). The ranges and nullspaces of $P_{I}$ and $Q_{J}$ have several interesting properties. First we need the following lemma:

Lemma 4.1. Let $P, Q$ be orthogonal projections such that $\|P-Q\|=1$. Then one and only one of the following conditions hold:

1. $N(P)+R(Q)=\mathcal{H}$, with non direct sum (and this is equivalent to $R(P)+N(Q)$ being a direct sum and a closed proper subspace of $\mathcal{H})$.

2. $R(P)+N(Q)=\mathcal{H}$, with non direct sum (and this is equivalent to $N(P)+R(Q)$ being a direct sum and a closed proper subspace of $\mathcal{H})$. 
3. $R(P)+N(Q)$ is non closed (and this is equivalent to $N(P)+R(Q)$ being non closed).

Proof. By the Krein-Krasnoselskii-Milman formula (see for instance [19])

$$
\|P-Q\|=\max \{\|P(1-Q)\|,\|Q(1-P)\|\},
$$

we have that one and only one of the following hold:

1. $\|P(1-Q)\|<1$ and $\|Q(1-P)\|=1$,

2. $\|P(1-Q)\|=1$ and $\|Q(1-P)\|<1$, or

3. $\|P(1-Q)\|=1$ and $\|Q(1-P)\|=1$.

This alternative corresponds precisely with the three conditions in the Lemma. It is known [9] that for two orthogonal projections $E$ and $F,\|E F\|<1$ holds if and only if $R(E) \cap R(F)=\{0\}$ and $R(E)+R(F)$ closed. The sum $\mathcal{M}+\mathcal{N}$ of two subspaces is closed if and only if the sum $\mathcal{M}^{\perp}+\mathcal{N}^{\perp}$ is closed (see [9]). Therefore, $\|E F\|<1$ is also equivalent to $N(E)+N(F)=\mathcal{H}$.

If we apply these facts to $E=P$ and $F=1-Q$, we obtain that the first alternative is equivalent to $R(P) \cap N(Q)=\{0\}$ and $R(P)+N(Q)$ closed, or to $N(P)+R(Q)=\mathcal{H}$.

Analogously, the second alternative is equivalent to $R(Q) \cap N(P)=\{0\}$ and $R(Q)+N(P)$ closed, or to $N(Q)+R(P)=\mathcal{H}$.

Note that in the first case, $R(P)+N(Q)$ is proper, otherwise its orthogonal complement would be $N(P) \cap R(Q)=\{0\}$, which together with the fact that $N(P)+R(Q)=\mathcal{H}$ (closed!), would lead us to the second alternative.

Analogously in the second alternative, $N(P)+R(Q)$ is proper.

If neither of these two happen, it is clear that neither $R(P)+N(Q)$ nor (equivalently) the sum of the orthogonals $N(P)+R(Q)$ is closed.

We have the following:

Theorem 4.2. Let $I, J \subset \mathbb{R}^{n}$ with finite Lebesgue measure. Then

1. $R\left(P_{I}\right)+R\left(Q_{J}\right)$ is a closed proper subset of $L^{2}\left(\mathbb{R}^{n}\right)$, with infinite codimension. The sum is direct $\left(R\left(P_{I}\right) \cap R\left(Q_{J}\right)=\{0\}\right)$.

2. $N\left(P_{I}\right)+N\left(Q_{J}\right)=L^{2}\left(\mathbb{R}^{n}\right)$, and the sum is not direct $\left(N\left(P_{I}\right) \cap N\left(Q_{J}\right)\right.$ is infinite dimensional).

3. $R\left(P_{I}\right)+N\left(Q_{J}\right)$ and $N\left(P_{I}\right)+R\left(Q_{J}\right)$ are proper dense subspaces of $L^{2}\left(\mathbb{R}^{n}\right)$, and $R\left(P_{I}\right) \cap$ $N\left(Q_{J}\right)=N\left(P_{I}\right) \cap R\left(Q_{J}\right)=\{0\}$.

Proof. By the cited result [9], two projections $P, Q$, satisfy that $R(P)+R(Q)$ is closed and $R(P) \cap R(Q)=\{0\}$ if and only if $\|P Q\|<1$. It is also known (see above, [13]) that $\left\|P_{I} Q_{J}\right\|<1$. The intersection of these spaces is, in our case (using the notation of the Halmos decomposition)

$$
R\left(P_{I}\right) \cap R\left(Q_{J}\right)=\mathcal{H}_{11}=\{0\} .
$$

As remarked above, Lenard proved that $\mathcal{H}_{11}=\mathcal{H}_{10}=\mathcal{H}_{01}=\{0\}$, and $\mathcal{H}_{00}$ is infinite dimensional. The orthogonal complement of this sum is

$$
\left(R\left(P_{I}\right)+R\left(Q_{J}\right)\right)^{\perp}=N\left(P_{I}\right) \cap N\left(Q_{J}\right)=\mathcal{H}_{00} .
$$


Thus the first assertion follows.

In our case $\left\|P_{I}-Q_{J}\right\|=1([13],[22])$ thus we may apply the above Lemma.

The first condition cannot happen:

$$
\left(N\left(P_{I}\right)+R\left(Q_{J}\right)\right)^{\perp}=R\left(P_{I}\right) \cap N\left(Q_{J}\right)=\mathcal{H}_{10}=\{0\} .
$$

By a similar argument, neither the second condition can happen. Thus $R\left(P_{I}\right)+R\left(Q_{J}\right)$ is non closed, and its orthogonal complement is trivial. Thus the second and third assertions follow.

Remark 4.3. It is known (see for instance [12]), that if $P, Q$ are projections with $P Q$ compact and $R(P) \cap R(Q)=\{0\}$, then

$$
\|P Q\|<1 .
$$

In [6], the second named author and A. Maestripieri studied the set of operators $T \in \mathcal{B}(\mathcal{H})$ which are of the form $T=P Q$. Among other properties, they proved that $T$ may have many factorizations, but there is a minimal factorization (called canonical factorization of $T$ ), namely

$$
T=P_{\overline{R(T)}} P_{N(T)^{\perp}}
$$

which satisfies that if $T=P Q$, then $R(T) \subset R(P)$ and $N(T)^{\perp} \subset R(Q)$ (or equivalently $N(Q) \subset N(T))$. Following this notation,

Proposition 4.4. The factorization $P_{I} Q_{J}$ is canonical.

Proof. Put $T=P_{I} Q_{J}$. Using Halmos decomposition in this particular case $\left(\mathcal{H}=\mathcal{H}_{00} \oplus(\mathcal{L} \times \mathcal{L})\right)$, apparently

$$
P_{I} Q_{J} P_{I}=0 \oplus\left(\begin{array}{cc}
C & 0 \\
0 & 0
\end{array}\right),
$$

and thus $R\left(P_{I} Q_{J} P_{I}\right)=0 \oplus(R(C) \times 0)$. Recall that $C^{2}>0$, and thus $C^{2}$ has dense range. It follows that

$$
\overline{R(T)}=\overline{R\left(P_{I} Q_{J}\right)}=\overline{R\left(P_{I} Q_{J} P_{I}\right)}=0 \oplus(\mathcal{L} \times 0),
$$

which is precisely the range of $P_{I}: \overline{R(T)}=R\left(P_{I}\right)$. Note the following elementary fact:

$$
N(P Q)=N(Q) \oplus(R(Q) \cap N(P)) .
$$

For the factorization $T=P_{I} Q_{J}$ it is known $([22])$ that $R\left(Q_{J}\right) \cap N\left(P_{I}\right)=0$. Thus

$$
N(T)=N\left(P_{I} Q_{J}\right)=N\left(Q_{J}\right)
$$

and the proof follows.

In [6] it is proven that if $T=P Q=P_{0} Q_{0}$, and the latter is the canonical factorization, then

$$
\left\|P_{0} f-Q_{0} f\right\| \leq\|P f-Q f\|
$$

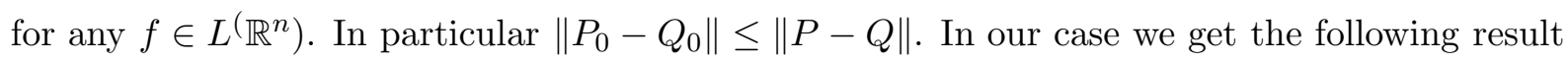
Corollary 4.5. Let $P, Q$ projections in $L^{2}\left(\mathbb{R}^{n}\right)$ such that $P Q=P_{I} Q_{J}$. Then for any $f \in L^{2}\left(\mathbb{R}^{n}\right)$ one has

$$
\left\|P_{I} f-Q_{J} f\right\|_{2} \leq\|P f-Q f\|_{2}
$$

In particular, $\left\|P_{I}-Q_{J}\right\| \leq\|P-Q\|$. 


\section{References}

[1] Amrein, W. O.; Sinha, K. B. On pairs of projections in a Hilbert space. Linear Algebra Appl. 208/209 (1994), 425-435.

[2] Andruchow, E.; Operators which are the difference of two projections. J. Math. Anal. Appl. 420 (2014), no. 2, 1634-1653.

[3] Arias, A.; Gudder, S. Almost sharp quantum effects. J. Math. Phys. 45 (2004), no. 11, 4196-4206.

[4] Berthier, A. M.; Jauch, J. M. A theorem on the support of functions in $L^{2}(\mathbf{R})$ and of their Fourier transforms. Lett. Math. Phys. 1 (1975/76), no. 2, 93-97.

[5] Böttcher, A.; Spitkovsky, I. M. A gentle guide to the basics of two projections theory. Linear Algebra Appl. 432 (2010), no. 6, 1412-1459.

[6] Corach, G.; Maestripieri, A. Products of orthogonal projections and polar decompositions. Linear Algebra Appl. 434 (2011), no. 6, 1594-1609.

[7] Corach, G.; Porta, H.; Recht, L. The geometry of spaces of projections in $C^{*}$-algebras. Adv. Math. 101 (1993), no. 1, 59-77.

[8] Davis, C. Separation of two linear subspaces. Acta Sci. Math. Szeged 19 (1958) 172-187.

[9] Deutsch, F. Best approximation in inner product spaces, Springer-Verlag, New York, 2001.

[10] Dixmier, J. Position relative de deux variétés linéaires fermées dans un espace de Hilbert. (French) Revue Sci. 86, (1948). 387-399.

[11] Donoho, D. L.; Stark, P. B. Uncertainty principles and signal recovery. SIAM J. Appl. Math. 49 (1989), no. 3, 906-931.

[12] Feshchenko, I. S. On closedness of the sum of $n$ subspaces of a Hilbert space. Ukrainian Math. J. 63 (2012), no. 10, 1566-1622.

[13] Folland, G. B.; Sitaram, A. The uncertainty principle: a mathematical survey. J. Fourier Anal. Appl. 3 (1997), no. 3, 207-238.

[14] Fuchs, W. H. J., On the magnitude of Fourier transforms. Proc. Intern. Math. Congr., Amsterdam, North-Holland, Amsterdam (1954).

[15] Gröchenig, K. Foundations of time-frequency analysis. Applied and Numerical Harmonic Analysis. Birkhuser Boston, Inc., Boston, MA, 2001.

[16] Halmos, P. R. Two subspaces. Trans. Amer. Math. Soc. 144 (1969) 381-389.

[17] Havin, V.; Jöricke, B. The uncertainty principle in harmonic analysis, Springer-Verlag, Berlin, 1994.

[18] Hogan, J. A.; Lakey, J. D. Time-frequency and time-scale methods. Adaptive decompositions, uncertainty principles, and sampling, Birkhäuser, Boston, 2005. 
[19] Krein, M. G. The theory of self-adjoint extensions of semi-bounded Hermitian transformations and its applications. II. (Russian) Mat. Sbornik N.S. 21(63), (1947). 365-404.

[20] Landau, H. J.; Pollak, H. O. Prolate spheroidal wave functions, Fourier analysis and uncertainty. II. Bell System Tech. J. 40 (1961), 65-84.

[21] Landau, H. J.; Pollak, H. O. Prolate spheroidal wave functions, Fourier analysis and uncertainty. III. The dimension of the space of essentially time- and band-limited signals. Bell System Tech. J. 41 (1962), 1295-1336.

[22] Lenard, A. The numerical range of a pair of projections. J. Functional Analysis 10 (1972), 410-423.

[23] Nees, M. Products of orthogonal projections as Carleman operators. Integral Equations Operator Theory 35 (1999), no. 1, 85-92.

[24] Porta, H.; Recht, L. Minimality of geodesics in Grassmann manifolds. Proc. Amer. Math. Soc. 100 (1987), no. 3, 464-466.

[25] Slepian, D.; Pollak, H. O. Prolate spheroidal wave functions, Fourier analysis and uncertainty. I. Bell System Tech. J. 40 (1961), 43-63.

[26] Smith, K. T., The uncertainty principle on groups, SIAM J. Appl. Math. 50 (1990), 876882.

(Esteban Andruchow) Instituto de Ciencias, Universidad Nacional de Gral. Sarmiento, J.M. Gutierrez 1150, (1613) Los Polvorines, Argentina and Instituto Argentino de Matemática, 'Alberto P. Calderón', CONICET, Saavedra 15 3er. piso, (1083) Buenos Aires, Argentina.

(Gustavo Corach) Instituto Argentino de Matemática, 'Alberto P. Calderón', CONICET, Saavedra 15 3er. piso, (1083) Buenos Aires, Argentina, and Depto. de Matemática, Facultad de Ingeniería, Universidad de Buenos Aires, Argentina. 